\title{
Spectral deformation for two-body dispersive systems with e.g. the Yukawa potential
}

\author{
Matthias Engelmann \\ Fachbereich Mathematik \\ Universität Stuttgart \\ Morten Grud Rasmussen \\ Department of Mathematical Sciences \\ Aalborg University
}

July 17, 2018

\begin{abstract}
We find an explicit closed formula for the $k$ 'th iterated commutator $\operatorname{ad}_{A}^{k}\left(H_{V}(\xi)\right)$ of arbitrary order $k \geq 1$ between a Hamiltonian $H_{V}(\xi)=M_{\omega_{\xi}}+S_{\breve{V}}$ and a conjugate operator $A=\frac{\mathrm{i}}{2}\left(v_{\xi} \cdot \nabla+\nabla \cdot v_{\xi}\right)$, where $M_{\omega_{\xi}}$ is the operator of multiplication with the real analytic function $\omega_{\xi}$ which depends real analytically on the parameter $\xi$, and the operator $S_{\check{V}}$ is the operator of convolution with the (sufficiently nice) function $\check{V}$, and $v_{\xi}$ is some vector field determined by $\omega_{\xi}$. Under certain assumptions, which are satisfied for the Yukawa potential, we then prove estimates of the form $\left\|\operatorname{ad}_{A}^{k}\left(H_{V}(\xi)\right)\left(H_{0}(\xi)+\mathfrak{i}\right)^{-1}\right\| \leq C_{\xi}^{k} k$ ! where $C_{\xi}$ is some constant which depends continuously on $\xi$. The Hamiltonian is the fixed total momentum fiber Hamiltonian of an abstract two-body dispersive system and the work is inspired by a recent result [3] which, under conditions including estimates of the mentioned type, opens up for spectral deformation and analytic perturbation theory of embedded eigenvalues of finite multiplicity.
\end{abstract}

Mathematical Subject Classification: 81Q10, 47B47

Keywords: dispersive systems, iterated commutators, spectral deformation, Yukawa potentials 


\section{Introduction}

In this paper we consider a two-body dispersive system where the two particles interact via a pair-potential $V$. We study the iterated commutators $\operatorname{ad}_{A}^{k}\left(H_{V}(\xi)\right)=$ $\left[\operatorname{ad}_{A}^{k-1}\left(H_{V}(\xi)\right), A\right]$ of the fixed total momentum fiber Hamiltonian $H_{V}(\xi)$ of this system with an operator $A$, which (in the sense of Mourre) is conjugate to $H_{V}(\xi)$. It is well-known from the literature that the nature of the spectrum and regularity of eigenstates is related to the (iterated) commutators with a conjugate operator, see e.g. [12, 9, 1, 8, 2, 4, 5, 11. See also [14] for another result where control of iterated commutators is needed.

Recently, together with Jacob Schach Møller, the authors developed an analytic perturbation theory for embedded eigenvalues in [3], which also contains an example of a non-trivial model which satisfies the needed conditions for the abstract theory of that paper. We consider a version of this model (introduced in details in Section 2) which has fiber Hamiltonians of the type

$$
H_{V}(\xi)=\omega_{\xi}+S_{\check{V}}
$$

where $\omega_{\xi}$ denotes multiplication by a certain analytic function (see Condition 2.1) and $S_{\breve{V}}$ denotes convolution by the inverse Fourier transform of the interaction potential $V$. The abstract conditions in [3] involve the requirement that there exists a constant $C_{\xi}>0$ such that for all $k \in \mathbb{N}$, the iterated commutator $\operatorname{ad}_{A}^{k}\left(H_{V}(\xi)\right)$ exists as a $H_{V}(\xi)$-bounded operator and $\left\|\operatorname{ad}_{A}^{k}\left(H_{V}(\xi)\right)\left(H_{V}(\xi)+\mathfrak{i}\right)^{-1}\right\| \leq C_{\xi}^{k} k$ !, which serves as a motivation to study these iterated commutators.

The result presented in this paper covers the results on the model in [3], but can deal with more singular potentials such as the Yukawa potential, see Proposition 2.7 The main result of this paper, Theorem 2.5. states the existence of a constant $C_{\xi}>0$ such that the bound on the iterated commutators mentioned above holds, given that $V$ satisfies either Condition 2.2 or Condition 2.4. In fact, we prove that this constant is continuous as a function of $\xi$. This allows us to reach the conclusion of [3, Theorem 3.2], if certain further assumptions are satisfied, namely relative boundedness of $S_{\breve{V}}$ wrt. the multiplication operator $\omega_{\xi}$ and that $\left[H_{V}(\xi), i A\right]$ satisfies a Mourre estimate. See Theorem 2.10 for details. We note that these further assumptions have already been verified for a large class of potentials in [3].

The main obstacle in proving the bound on the iterated commutator is to control the commutator with the interaction term. This is done in several steps. First, we find a closed formula for the $k$ 'th iterated commutator for any $k$, see Theorem 4.1 . Then we find appropriate estimates for every term that appears in the formula. These bounds then turn out to have the right behaviour.

The paper is organized as follows. In Section 2 the model is introduced, conditions are stated, and the main results are formulated. To write the closed commutator formula in a compact, readable form and for use in proofs and various intermediate results, we introduce some terminology and notation in Section 3 . In Section 4, we then state and prove the main technical result, Theorem 4.1, which is the closed commutator formula. In Section 5, a technical lemma is proven which paves the way for an estimate 
on the scalar factors in the formula, which is then stated and proved in Section 6. The next step is to estimate the number of terms in the sum in the formula, which is done in Section 7 Before stating some sufficient a posteriori assumptions and making the final estimates on the interaction commutator, we turn our attention to the iterated commutator of the free Hamiltonian in Section 8. This section is essentially a repetition of known results by the present authors and J. S. Møller, see [3], and is included for the reader's convenience. The methods used for the free Hamiltonian can be copied to deal with certain parts of the interaction commutator, which we return to in Section 9 . where we identify a posteriori assumptions which are sufficient to prove the right kind of bounds on the iterated commutators with the interaction term. The main result in this section is Theorem 9.4 in which we show that Conditions 2.2 and 2.4 both lead to the desired bounds. We conclude with Section 10 where Proposition 2.7, which states that in dimension $d=3$ Yukawa potentials satisfy Condition 2.2 is proven.

\section{The model and results}

We introduce a two-particle Hamiltonian on $\mathrm{L}^{2}\left(\mathbb{R}^{2 d}\right)$ by

$$
H_{V}^{\prime}=\omega_{1}\left(p_{1}\right)+\omega_{2}\left(p_{2}\right)+V\left(x_{1}-x_{2}\right)
$$

where $p_{i}=-\mathfrak{i} \nabla_{x_{i}}, x_{i} \in \mathbb{R}^{d}$.

We impose the following set of conditions on $\omega_{1}, \omega_{2}$ and $V$ :

Condition 2.1 (Properties of $\omega_{1}, \omega_{2}$ and $V$ ).

1. The $\omega_{i}$ 's are real-valued, real analytic functions on $\mathbb{R}^{d}$ and there exists $R>0$, such that the $\omega_{i}$ 's extend to analytic functions in the d-dimensional strip

$$
S_{2 R}^{d}:=\left\{\left(z_{1}, \ldots, z_{d}\right) \in \mathbb{C}^{d}|| \operatorname{Im}\left(z_{i}\right) \mid<2 R, i=1, \ldots, d\right\} .
$$

We denote the analytic continuations of these functions by the same symbols.

2. There exist real numbers $p=s_{2} \geq s_{1}, s_{2}>0$ and a constant $C>0$ such that

$$
\left|\partial^{\alpha} \omega_{j}(k)\right| \leq C\langle k\rangle^{s_{j}}, \quad\left|\omega_{j}(k)\right| \geq \frac{1}{C}\langle k\rangle^{s_{j}}-C
$$

for every multi-index $\alpha \in \mathbb{N}_{0}^{d},|\alpha| \leq 1$ and all $k \in S_{2 R}^{d}$.

3. The Fourier transform $\hat{V}$ of $V$ exists and $\hat{V} \in \mathrm{L}_{\text {loc }}^{1}\left(\mathbb{R}^{d}\right)$.

Here and hereafter, $\langle x\rangle=\sqrt{x^{2}+1}$ and $\hat{f}=f^{\wedge}$ denotes the Fourier transform of $f$. Conjugating with the Fourier transform, we see that $H_{V}^{\prime}$ is unitarily equivalent to

$$
H_{V}=\omega_{1}\left(k_{1}\right)+\omega_{2}\left(k_{2}\right)+t_{V}
$$

where $t_{V}$ is the partial convolution operator

$$
\left(t_{V} f\right)\left(k_{1}, k_{2}\right):=\int_{\mathbb{R}^{d}} \hat{V}(u) f\left(k_{1}-u, k_{2}+u\right) \mathrm{d} u
$$


and

$$
\hat{V}(k)=(2 \pi)^{-d / 2} \int_{\mathbb{R}^{d}} \mathrm{e}^{-\mathfrak{i} k \cdot x} V(x) \mathrm{d} x .
$$

In order to fibrate $H_{V}$ w.r.t. total momentum $\xi=k_{1}+k_{2}$, we introduce a unitary operator $I: \mathrm{L}^{2}\left(\mathbb{R}^{d} \times \mathbb{R}^{d}\right) \rightarrow \mathrm{L}^{2}\left(\mathbb{R}^{d} ; L^{2}\left(\mathbb{R}^{d}\right)\right)$ by setting

$$
(I f)(\xi)=f(\xi-\cdot, \cdot) .
$$

Under this transformation, we find that the Hamiltonian takes the form

$$
I H_{V} I^{*}=\int_{\mathbb{R}^{d}}^{\oplus} H_{V}(\xi) \mathrm{d} \xi, \quad \text { where } \quad H(\xi)=H_{V}(\xi)=\omega_{\xi}+S_{\check{V}},
$$

and

$$
\omega_{\xi}(k)=\omega_{1}(\xi-k)+\omega_{2}(k), \quad\left(S_{\check{V}} f\right)(k)=(\check{V} * f)(k) .
$$

Here $\check{V}(k)=\hat{V}(-k)$ is the inverse Fourier transform of $V$ and $\check{V} * f$ denotes the convolution product. Note that $H_{0}(\xi)$ corresponds to the case where the potential is absent. For brevity, we will often suppress the subscript $V$ in $H_{V}(\xi)$ and just write $H(\xi)$. For later use, let $T_{0}=S_{\breve{V}}$ for a fixed $V$.

Furthermore, we define a self-adjoint operator for every total momentum $\xi \in \mathbb{R}^{d}$ by

$$
A_{\xi}=\frac{\mathfrak{i}}{2}\left(v_{\xi} \cdot \nabla_{k}+\nabla_{k} \cdot v_{\xi}\right) .
$$

If no confusion can arise, we will just write $A=A_{\xi}$. The vector field $v_{\xi}$ is given by

$$
v_{\xi}(k)=\mathrm{e}^{-k^{2}-\xi^{2}}\left(\nabla_{k} \omega_{\xi}\right)(k) .
$$

The choice of vector field can be regarded as a generalization of the standard choice $A=\frac{\mathfrak{i}}{2} x \cdot \nabla+\frac{\mathfrak{i}}{2} \nabla \cdot x$ in Mourre theory for Schrödinger operators. Note that $\frac{1}{2} x=\nabla x^{2}$. Thus, the quadratic dispersion relation in the Schrödinger case gets substituted by a more general one. The exponential weight in both $x$ and $\xi$ is added to make the uniform estimates in Sections 8 and 9 work.

In addition to Condition 2.1, which is always assumed, we will sometimes need one of the following two conditions. First, we recall the definition of the weak $\mathrm{L}^{s}\left(\mathbb{R}^{d}\right)$ norm: $\|f\|_{s, w}=\sup _{\alpha>0}|\{x|| f(x) \mid>\alpha\}|^{\frac{1}{s}}$. Here $|A|$ for a subset $A$ of $\mathbb{R}^{d}$ denotes the Lebesgue measure of $A$. $\mathrm{L}_{w}^{s}\left(\mathbb{R}^{d}\right)$ is then the set of all functions for which $\|f\|_{s, w}<\infty$.

Condition 2.2 (L $\mathrm{L}_{w}^{s}$-bounds on $\left.\hat{V}\right)$. Let $s>1$ with $\max \left\{\frac{1}{2}, 1-\frac{p}{d}\right\}<\frac{1}{s} \leq \frac{2}{d}$, where $p=s_{2}$ comes from Condition 2.1. There exists $c^{\prime}>0$ such that

$$
\forall \alpha \in \mathbb{N}_{0}^{d}:\left\|\hat{V}^{(\alpha)}\right\|_{s, w} \leq \alpha ! c^{\prime|\alpha|},
$$

where $\|\cdot\|_{s, w}$ denotes the weak $\mathrm{L}^{s}\left(\mathbb{R}^{d}\right)$ norm.

Remark 2.3. Note that $\frac{1}{s} \leq \frac{2}{d}$ is only a restriction for $d \geq 3$; for lower dimensions the condition $s>1$ implies the former. 
Condition 2.4 ( $\mathrm{L}^{1}$-bounds on $\left.\hat{V}\right)$. There exists $c^{\prime}>0$ such that

$$
\forall \alpha \in \mathbb{N}_{0}^{d}:\left\|\hat{V}^{(\alpha)}\right\|_{1} \leq \alpha ! c^{|\alpha|}
$$

Theorem 9.4 can be proven for a potential satisfying Condition 2.2 or 2.4. However it is Condition 2.2 that we show to hold in the case of the Yukawa potential in Proposition 2.7

The main result of the paper is now as follows.

Theorem 2.5. Assume Condition 2.1 and either Condition 2.2 or Condition 2.4. Then there exists a constant $C(\xi)$ which depends continuously on $\xi$, such that

$$
\left\|\operatorname{ad}_{A}^{k}(H(\xi))\left(H_{0}(\xi)+\mathfrak{i}\right)^{-1}\right\| \leq C(\xi)^{k} k !
$$

for all $k \in \mathbb{N}$.

Proof. This follows directly from Proposition 8.1 and Theorem 9.4.

Remark 2.6. In fact, Theorem 2.5 is true under a weaker assumption which can be found in Theorem 9.4. The logical structure of the argument is that both Condition 2.2 and Condition 2.4 imply this weaker assumption.

The following Proposition implies that Condition 2.2 is satisfied for the Yukawa potential whenever $p>1$ and $\frac{3}{2} \leq s<2$.

Proposition 2.7. Let $d=3, V(x)=\frac{\mathrm{e}^{-|x|}}{|x|}$ and $s \geq 3 / 2$. Then $\hat{V}(k)=4 \pi\left(1+k^{2}\right)^{-1}$ and there exists $c>0$ such that

$$
\forall \alpha \in \mathbb{N}_{0}^{3}:\left\|\hat{V}^{(\alpha)}\right\|_{s, w} \leq \alpha ! c^{|\alpha|} .
$$

Remark 2.8. Some authors use the Fourier transform $\hat{V}(k)=4 \pi\left(1+k^{2}\right)^{-1}$ to define the Yukawa potential in other dimensions. In that case, a similar result holds in these dimensions.

Remark 2.9. In [3], the conclusion of Theorem 2.5] is (indirectly) reached by completely different arguments for the class of Hamiltonians satisfying the following condition in addition to Condition 2.1

- Let $d^{\prime}=2[d / 2]+2$. We suppose that $V \in C^{d^{\prime}}\left(\mathbb{R}^{d}\right)$ and there exists $a>0$, such that for all $\alpha \in \mathbb{N}_{0}^{d}$ with $|\alpha| \leq d^{\prime}$, we have $\sup _{x \in \mathbb{R}^{d}} \mathrm{e}^{a|x|}\left|\partial_{x}^{\alpha} V(x)\right|<\infty$.

It is easy to see that for dispersion relations and potentials satisfying these conditions, Condition 2.4 is also satisfied. Indeed, one can prove that for some $a>0, \hat{V}$ has an analytic continuation to the $d$-dimensional strip $S_{a}^{d}$ and

$$
\forall k \in S_{a}^{d}: \quad|\widehat{V}(k)| \leq C_{V}\left(1+|k|^{d^{\prime}}\right)^{-1}
$$


see Remark 3.4.4 in [3]. Then, in the case $d=1$,

$$
\begin{aligned}
\frac{2 \pi}{n !}\left\|\hat{V}^{(n)}\right\|_{1} & \leq \int\left(\int_{\Gamma_{r}} \frac{|\hat{V}(w)|}{|w-z|^{n+1}} \mathrm{~d} w\right) \mathrm{d} z \\
& \leq \int\left(\int_{\tilde{\Gamma}_{r}} \frac{C_{V}}{1+\left.|r-| z\right|^{2}} \frac{1}{r^{n+1}} \mathrm{~d} w\right) \mathrm{d} z \\
& =\frac{2 C_{V}}{r^{n}} \int \frac{\mathrm{d} z}{1+|r-| z||^{2}},
\end{aligned}
$$

where $\Gamma_{r}$ is a path around $z$ with radius $r<a$ and $\tilde{\Gamma}_{r}$ is a path around 0 . The case $d>1$ is similar.

In order to apply the abstract spectral deformation theory of $[3$, in addition to Theorem 2.5, we need to assume a few extra conditions which are clearly satisfied for the Yukawa potential and $p>1$, namely relative compactness of the interaction term and the existence of a Mourre estimate.

Theorem 2.10. Suppose Condition 2.1] and either Condition 2.2 or Condition 2.4. Assume that $T_{0}=S_{\breve{V}}$ is relatively bounded wrt. $H_{0}$ and that for all $\xi \in \mathbb{R}^{d}$ and all $\lambda \in \mathbb{R}$, there exists positive constants $e, C, \kappa>0$ and a compact operator $K$ such that

$$
\left[H(\xi), \mathfrak{i} A_{\xi}\right] \geq e-C E_{H(\xi)}(\mathbb{R} \backslash[\lambda-\kappa, \lambda+\kappa])\langle H(\xi)\rangle-K .
$$

Let $\Sigma_{\mathrm{pp}}$ be the joint energy-momentum point spectrum

$$
\Sigma_{\mathrm{pp}}=\left\{(\lambda, \xi) \in \mathbb{R} \times \mathbb{R}^{d} \mid \lambda \in \Sigma_{\mathrm{pp}}(\xi)\right\}, \quad \Sigma_{\mathrm{pp}}(\xi)=\sigma_{\mathrm{pp}}(H(\xi))
$$

and $\mathcal{T}$ the energy-momentum threshold set

$$
\begin{aligned}
\mathcal{T} & =\left\{(\lambda, \xi) \in \mathbb{R} \times \mathbb{R}^{d} \mid \lambda \in \mathcal{T}(\xi)\right\}, \\
\mathcal{T}(\xi) & =\left\{\lambda \in \mathbb{R} \mid \exists k \in \mathbb{R}^{d}: \omega_{\xi}(k)=\lambda \text { and } \nabla_{k} \omega_{\xi}(k)=0\right\} .
\end{aligned}
$$

Let $\left(\lambda_{0}, \xi_{0}\right) \in \Sigma_{\mathrm{pp}} \backslash \mathcal{T}$. If we fix $v \in \mathbb{R}^{d}$ with $\|v\|=1$, then there exist

- $r, \rho>0$

- natural numbers $0 \leq m_{ \pm} \leq n_{0}$ and $n_{1}^{ \pm}, \ldots, n_{m_{ \pm}}^{ \pm} \geq 1$ with $n_{1}^{ \pm}+\cdots+n_{m_{ \pm}}^{ \pm} \leq n_{0}$,

- real analytic functions $\lambda_{1}^{ \pm}, \ldots, \lambda_{m_{ \pm}}^{ \pm}: I_{ \pm} \rightarrow \mathbb{R}, I_{-}=(-r, 0)$ and $I_{+}=(0, r)$,

such that

1. for any $j, \lim _{t \rightarrow 0 \pm} \lambda_{j}^{ \pm}(t)=\lambda_{0}$,

2. for any $t \in I_{ \pm}$, we have $\sigma_{\mathrm{pp}}\left(H\left(\xi_{0}+t v\right)\right) \cap\left(\lambda_{0}-\rho, \lambda_{0}+\rho\right)=\left\{\lambda_{1}^{ \pm}(t), \ldots, \lambda_{m_{ \pm}}^{ \pm}(t)\right\}$,

3. The eigenvalue branches $I_{ \pm} \ni t \rightarrow \lambda_{j}^{ \pm}(t)$ have constant multiplicity $n_{j}^{ \pm}$.

This theorem, with the assumptions replaced by just Condition 2.1] and the condition mentioned in Remark 2.9] is similar to [3, Theorem 3.2]. This result, however, doesn't cover the Yukawa potential due to the singularity at $x=0$. 


\section{Terminology and notation}

In this section, we introduce some notation which we use to state and prove Theorem 4.1. The main objects are the polyindices which we usually denote $\alpha, \beta, a$ or $b$ (see below), and the two classes of operators indexed by the polyindices; the multiplication operators $M_{\alpha, \beta}$ and the convolution operators $T_{\beta, b}$.

In the following, $C_{0}\left(\mathbb{N}_{0}, \mathbb{N}_{0}^{d}\right)$ will denote the set of non-negative, integer valued functions on $\mathbb{N}_{0}$ which are zero except on a finite set. Such functions will be referred to as polyindices of dimension $d$. We will usually use the letters $\alpha$ and $a$ for 1dimensional polyindices, while $\beta$ and $b$ are reserved for $d$-dimensional polyindices. For any polyindex $\beta \in C_{0}\left(\mathbb{N}_{0}, \mathbb{N}_{0}^{d}\right)$ of dimension $d$, we will call the finite number

$$
\|\beta\|=\sum_{\sigma=1}^{d} \sum_{i=0}^{\infty} \beta_{\sigma}(i)(i+1)
$$

the order of $\beta$, and the finite number

$$
|\beta|=\sum_{\sigma=1}^{d} \sum_{i=0}^{\infty} \beta_{\sigma}(i)
$$

will be called the size of $\beta$. The order factorial of $\beta$ will be written as, and defined by,

$$
\beta \ddot{!}=\prod_{\sigma=1}^{d} \prod_{i=0}^{\infty} \beta_{\sigma}(i) !((i+1) !)^{\beta_{\sigma}(i)},
$$

and is likewise a finite number for any polyindex $\beta$ (all factors except a finite number are 1). The reduced order factorial of $\beta$ is then

$$
\beta !=\prod_{\sigma=1}^{d} \prod_{i=0}^{\infty} \beta_{\sigma}(i) !(i+1)^{\beta_{\sigma}(i)} .
$$

We will sometimes need the ratio $\beta \overline{!}:=\frac{\beta !}{\beta !}=\prod_{\sigma=1}^{d} \prod_{i=0}^{\infty}(i !)^{\beta_{\sigma}(i)}$.

Remark 3.1. At a later point in the paper we will need the following result which is easily checked to be true.

$$
\left|\left\{\alpha \in \mathrm{C}_{0}\left(\mathbb{N}_{0}, \mathbb{N}_{0}\right) \mid\|\alpha\|=k\right\}\right|=p(k),
$$

where $p(k)$ is the number theoretic partition function (see e.g. [6]) and $|M|$ denotes the number of elements of a set $M$. Indeed, if $\|\alpha\|=k$, then $\alpha$ determines a unique partition of $k$ in the following way:

$$
k=\|\alpha\|=\sum_{i=0}^{\infty} \sum_{j=1}^{\alpha(i)}(i+1),
$$

and if a partition of $k$ is given, it can be uniquely encoded in an $\alpha$ with $\|\alpha\|=k$ by letting $\alpha(i-1)$ denote the number of $i$ 's in the partition for all $i \geq 1$. A simple upper bound for $p(k)$ is $p(k)<e^{\pi \sqrt{2 k / 3}}=e^{c \sqrt{k}}$, cf. [6]. 
The polyindices will be used to index certain operators that appear in the commutator formula for the interaction. More specifically, let $\alpha$ and $\beta$ be a polyindices of dimension 1 and $d$, respectively, and let $f \in C^{\infty}(\mathbb{R}, \mathbb{R})$ and $g \in C^{\infty}\left(\mathbb{R}^{d}, \mathbb{R}\right)$. Write

$$
\mathbb{D}_{v} f^{\alpha}=\prod_{i=0}^{\infty}\left(D_{v}^{i} f\right)^{\alpha(i)} \quad \text { and } \quad \mathbb{D}_{v} g^{\beta}=\prod_{i=0}^{\infty} \prod_{\sigma=1}^{d}\left(D_{v}^{i} g_{\sigma}\right)^{\beta_{\sigma}(i)} \quad \text { where } \quad D_{v}=\mathfrak{i} v_{\xi} \cdot \nabla .
$$

We define

$$
M_{\alpha, \beta}:=M_{\mathbb{D}_{v} w^{\alpha} \mathbb{D}_{v} v_{\xi}^{\beta}},
$$

where $w=\frac{\mathfrak{i}}{2} \operatorname{div}\left(v_{\xi}\right)$ and, for a function $f, M_{f}$ denotes the operator of multiplication with $f$. We note that $M_{0,0}=\mathbb{1}$ and that $M_{\alpha, \beta} M_{a, b}=M_{\alpha+a, \beta+b}$.

The commutator formula also contains some convolution operators which are indexed by polyindices. For any pair of $d$-dimensional polyindices $\beta$ and $b$, we now let the $d$-dimensional multiindex $\gamma_{\beta+b}$ be defined through

$$
\left(\gamma_{\beta+b}\right)_{\sigma}=\left|\beta_{\sigma}\right|+\left|b_{\sigma}\right| .
$$

Then

$$
T_{\beta, b}:=S_{\left((-1)^{|b|}(-x)^{\gamma_{\beta+b}} \bar{V}\right)^{\wedge},}
$$

where $S_{f}$ denotes the operator of convolution with the function $f$ and $\bar{V}(x)=V(-x)$. Note that, although only $\hat{V}$ is assumed to be well-defined, $T_{\beta, b}$ can be interpreted as a form on $C_{0}^{\infty}\left(\mathbb{R}^{d}\right)$ for all $\beta$ and $b$. Observe that $S_{\hat{\bar{V}}}=T_{0}=T_{0,0}$.

In the proof of the commutator formula, we will make extensive use of the following notation. If $\gamma \in \mathbb{N}_{0}^{d}$ is a multiindex and $\sigma \in\{1, \ldots, d\}$, then we let

$$
\gamma^{+(\sigma)}=\gamma+\delta_{\sigma}
$$

where $\delta_{\sigma}$ is given by $\left(\delta_{\sigma}\right)_{\sigma^{\prime}}=\delta_{\sigma, \sigma^{\prime}}$, where $\delta_{\sigma, \sigma^{\prime}}$ is the Kronecker delta. For a 1dimensional polyindex $\alpha \in C_{0}\left(\mathbb{N}_{0}, N_{0}\right)$, we define

$$
\alpha^{+(i)}=\alpha+\delta_{i},
$$

where $\delta_{i}(j)=\delta_{i, j}$. If $\beta \in C_{0}\left(\mathbb{N}_{0}, \mathbb{N}_{0}^{d}\right)$ is a $d$-dimensional polyindex, then

$$
\beta^{+(i, \sigma)}=\beta+\delta_{(i, \sigma)},
$$

where $\left(\delta_{(i, \sigma)}(j)\right)_{\sigma^{\prime}}=\delta_{i, j} \delta_{\sigma, \sigma^{\prime}}$. Likewise, we will write

$$
\alpha^{-(i)}=\alpha-\delta_{i} \quad \text { and } \quad \beta^{-(i, \sigma)}=\beta-\delta_{(i, \sigma)},
$$

whenever $\alpha(i), \beta_{\sigma}(i) \geq 1$. If $\alpha(i)$ or $\beta_{\sigma}(i)$ is 0 , then $\alpha^{-(i)}$ respectively $\beta^{-(i, \sigma)}$ can be given any (1-respectively $d$-dimensional polyindex) value; such cases will only appear in expressions that are multiplied by 0 . 


\section{The commutator formula for the interaction term}

Theorem 4.1. Let $T_{0}=S_{\breve{V}}$ denote the interaction term. Then

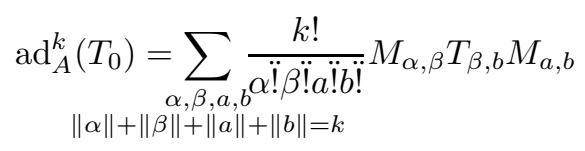

as a form identity on $C_{0}^{\infty}\left(\mathbb{R}^{d}\right)$.

Proof. The proof goes by induction. The case $k=0$ is trivially true. Consider

$$
\left[A, M_{\alpha, \beta} T_{\beta, b} M_{a, b}\right]=\left[A, M_{\alpha, \beta}\right] T_{\beta, b} M_{a, b}+M_{\alpha, \beta}\left[A, T_{\beta, b}\right] M_{a, b}+M_{\alpha, \beta} T_{\beta, b}\left[A, M_{a, b}\right],
$$

which here and for the rest of this section should be read as form identities on $C_{0}^{\infty}\left(\mathbb{R}^{d}\right)$. We thus need to find $\left[A, M_{\alpha, \beta}\right]$ and $\left[A, T_{\beta, b}\right]$ :

$$
\left[A, M_{\alpha, \beta}\right]=\sum_{i=0}^{\infty}\left(\alpha(i) M_{\alpha^{-(i)+(i+1), \beta}}+\sum_{\sigma=1}^{d} \beta_{\sigma}(i) M_{\alpha, \beta^{-(i, \sigma)+(i+1, \sigma)}}\right)
$$

while

$$
\left(A T_{\beta, b} f\right)(k)=\left(M_{w_{\xi}} T_{\beta, b} f\right)(k)+\sum_{\sigma=1}^{d}\left(M_{\left(v_{\xi}\right)_{\sigma}} T_{\beta+(0, \sigma), b} f\right)(k)
$$

and

$$
\left(T_{\beta, b} A f\right)(k)=-\left(T_{\beta, b} M_{w_{\xi}} f\right)(k)-\sum_{\sigma=1}^{d}\left(T_{\beta, b^{+(0, \sigma)}} M_{\left(v_{\xi}\right)_{\sigma}} f\right)(k),
$$

so

$$
\left[A, T_{\beta, b}\right]=M_{\delta_{0}, 0} T_{\beta, b}+T_{\beta, b} M_{\delta_{0}, 0}+\sum_{\sigma=1}^{d}\left(M_{0, \delta_{(0, \sigma)}} T_{\beta+(0, \sigma), b}+T_{\beta, b+(0, \sigma)} M_{0, \delta_{(0, \sigma)}}\right) .
$$

Putting this together (and noting that $T_{\beta, b}=T_{\beta^{-(i, \sigma)+(i+1, \sigma), b}}=T_{\beta, b^{-(i, \sigma)+(i+1, \sigma)}}$ ), we get

$$
\begin{aligned}
& {\left[A, M_{\alpha, \beta} T_{\beta, b} M_{a, b}\right]=} \\
& \sum_{i=0}^{\infty}\left(\alpha(i) M_{\alpha^{-(i)+(i+1), \beta}} T_{\beta, b} M_{a, b}+\sum_{\sigma=1}^{d} \beta_{\sigma}(i) M_{\alpha, \beta^{-(i, \sigma)+(i+1, \sigma)}} T_{\beta^{-(i, \sigma)+(i+1, \sigma), b}} M_{a, b}\right) \\
& +M_{\alpha+(0), \beta} T_{\beta, b} M_{a, b}+M_{\alpha, \beta} T_{\beta, b} M_{a^{+(0)}, b} \\
& +\sum_{\sigma=1}^{d}\left(M_{\alpha, \beta+(0, \sigma)} T_{\beta^{+(0, \sigma), b}} M_{a, b}+M_{\alpha, \beta} T_{\beta, b^{+(0, \sigma)}} M_{a, b^{+(0, \sigma)}}\right) \\
& +\sum_{i=0}^{\infty}\left(a(i) M_{\alpha, \beta} T_{\beta, b} M_{a^{-(i)+(i+1)}, b}+\sum_{\sigma=1}^{d} b_{\sigma}(i) M_{\alpha, \beta} T_{\beta, b^{-(i, \sigma)+(i+1, \sigma)}} M_{a, b^{-(i, \sigma)+(i+1, \sigma)}}\right) .
\end{aligned}
$$


From this expression and the induction start, it is clear that the $k^{\prime}$ th iterated commutator is of the form

$$
\begin{gathered}
\operatorname{ad}_{A}^{k}\left(T_{0}\right)=\sum_{\substack{\alpha, \beta, a, b \\
\|\alpha\|+\|\beta\|+\|a\|+\|b\|=k}} C_{\alpha, \beta, a, b}^{(k)} M_{\alpha, \beta} T_{\beta, b} M_{a, b}, \\
\end{gathered}
$$

where $C_{\alpha, \beta, a, b}^{(k)}$ are some constants to be determined. Assume that the commutator formula holds true for $k$. We want to show that it also holds for $k+1$. By the above discussion, it is enough to let $\alpha, \beta, a, b$ be arbitrary with $\|\alpha\|+\|\beta\|+\|a\|+\|b\|=k+1$ and show that $C_{\alpha, \beta, a, b}^{(k)}=\frac{k !}{\alpha ! \beta ! a ! b !}$, so this is what we do.

Using the induction hypothesis we combine (6) and (7) to obtain an expression for $\operatorname{ad}_{A}^{k+1}\left(T_{0}\right)$. This enables us to identify those terms in the $k$ 'th iterated commutator that through commutation with $A$ contribute to the term $C_{\alpha, \beta, a, b}^{(k+1)} M_{\alpha, \beta} T_{\beta, b} M_{a, b}$ in the $k+1$ 'st iterated commutator. Before proceeding we illustrate this by an example. Suppose we are given $\alpha, \beta, a, b$ with $\|\alpha\|+\|\beta\|+\|a\|+\|b\|=k+1$. One of the terms appearing in the combination of (6) and (7) is

$$
C_{\alpha^{\prime}, \beta^{\prime}, a^{\prime}, b^{\prime}}^{(k)} \alpha^{\prime}(i) M_{\alpha^{\prime-(i)+(i+1), \beta^{\prime}}} T_{\beta^{\prime}, b^{\prime}} M_{a^{\prime}, b^{\prime}},
$$

where

$$
\left\|\alpha^{\prime}\right\|+\left\|\beta^{\prime}\right\|+\left\|a^{\prime}\right\|+\left\|b^{\prime}\right\|=k .
$$

Since the contributing term from the $k$ 'th commutator can only have one polyindex deviating from $\alpha, \beta, a, b$, this term will contribute to $C_{\alpha, \beta, a, b}^{(k+1)} M_{\alpha, \beta} T_{\beta, b} M_{a, b}$, if $\alpha^{\prime}=$ $\alpha^{+(i)-(i+1)}, \beta^{\prime}=\beta, a^{\prime}=a$ and $b^{\prime}=b$. In the same fashion one easily finds that all possible contributors have polyindices of one of the following forms:

$$
\begin{array}{llll}
\left(\alpha^{-(0)}, \beta, a, b\right), & \left(\alpha^{-(i+1)+(i)}, \beta, a, b\right), & \left(\alpha, \beta^{-(0, \sigma)}, a, b\right), & \left(\alpha, \beta^{-(i+1, \sigma)+(i, \sigma)}, a, b\right), \\
\left(\alpha, \beta, a^{-(0)}, b\right), & \left(\alpha, \beta, a^{-(i+1)+(i)}, b\right), & \left(\alpha, \beta, a, b^{-(0, \sigma)}\right), & \left(\alpha, \beta, a, b^{-(i+1, \sigma)+(i, \sigma)}\right) .
\end{array}
$$

Appealing again to the induction hypothesis and (6), we see that in our example

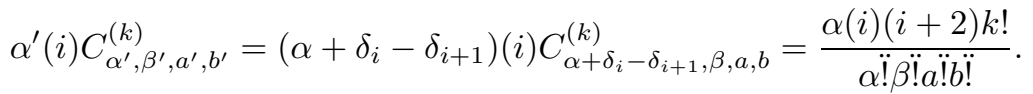

Proceeding in the same way we see that the contributions of all the previously listed terms are, respectively

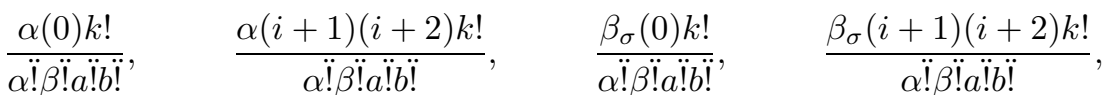

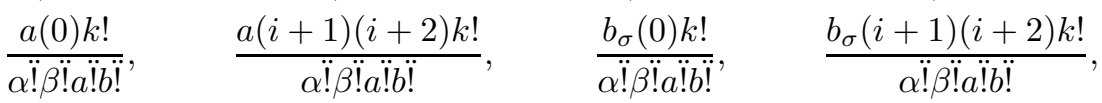

times $M_{\alpha, \beta} T_{\beta, b} M_{a, b}$. Finally, summing up all these possible contributions gives us the 
value of $C_{\alpha, \beta, a, b}^{(k+1)}$ :

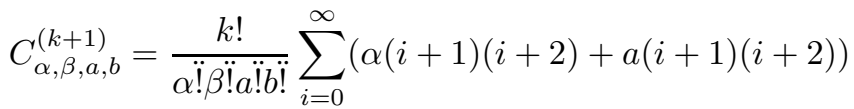

$$
\begin{aligned}
& +\frac{k !}{\alpha ! \beta \ddot{!} a ! b !} \sum_{i=0}^{\infty} \sum_{\sigma=1}^{d}\left(\beta_{\sigma}(i+1)(i+2)+b_{\sigma}(i+1)(i+2)\right)
\end{aligned}
$$

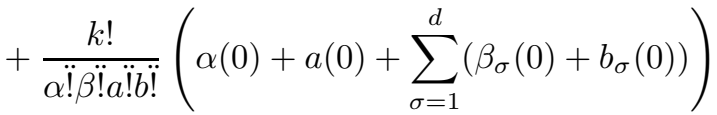

$$
\begin{aligned}
& =\frac{k !}{\alpha \ddot{!} \beta \ddot{a} a \ddot{!} b \ddot{!}}(\|\alpha\|+\|a\|+\|\beta\|+\|b\|)=\frac{(k+1) !}{\alpha \ddot{!} \beta \ddot{!} a \ddot{!} b \ddot{!}},
\end{aligned}
$$

where we have used that by assumption $\|\alpha\|+\|a\|+\|\beta\|+\|b\|=k+1$ in the last line. This completes the proof.

\section{Estimates on the reduced order factorial of certain polyindices}

To be able to use Theorem 4.1 and (5) to estimate $\operatorname{ad}_{A}^{k}\left(T_{0}\right)$, we need some control on the reduced order factorials, $\alpha$ !, $\beta$ !, etc. In fact, we will just use the trivial estimates $a !, \alpha ! \geq 1$ for the reduced order factorial of the 1-dimensional polyindices pertaining to the multiplication operators of the form $M_{\mathbb{D}_{v} w^{\alpha}}$.

For the $d$-dimensional polyindices $\beta$ and $b$, we will make a more careful estimate. More precisely, for each $\sigma \in\{1,2, \ldots, d\}$, we want to estimate

$$
C^{\left\|\beta_{\sigma}\right\|+\left\|b_{\sigma}\right\|} \beta_{\sigma} ! b_{\sigma} !=\prod_{i=1}^{\infty}\left(\left(\prod_{y=1}^{\beta_{\sigma}(i)} C^{i+1} y(i+1)\right)\left(\prod_{y=1}^{b_{\sigma}(i)} C^{i+1} y(i+1)\right)\right)
$$

from below, where $C>0$ is some constant (see the next section for a "derivation" of the identity). We will use the factorization (8) and split the estimate into two parts. The first part is a careful estimate on the product of the smallest factors in (8), the last will be a rough estimate on the rest of the factors.

The first part part is contained in the following technical lemma, the rest is postponed to the next section.

Lemma 5.1. For any $C>0$ and $\ell \in \mathbb{N}$, let $\beta^{\ell}: \mathbb{N}_{0} \rightarrow \mathbb{N}_{0}$ denote the polyindex given by

$$
\beta^{\ell}(i)=\left\lfloor\frac{\ell}{C^{i}(i+1)}\right\rfloor,
$$

where $\lfloor x\rfloor$ denotes the integer part of $x$. For any $0<\varepsilon<1$, there exists a $C_{0}$ such that for all $C>C_{0}$ and all $\ell$,

$$
\left|\beta^{\ell}\right| \leq(1+\varepsilon) \ell
$$


and

$$
C^{2\left\|\beta^{\ell}\right\|}\left(\beta^{\ell !} !\right)^{2} \geq C^{\prime \prime 2\left|\beta^{\ell}\right|}\left(2\left|\beta^{\ell}\right|\right) !
$$

where $C^{\prime \prime}=C /(4 e(1+\varepsilon))$.

Proof. The size $\left|\beta^{\ell}\right|$ of $\beta^{\ell}$ clearly depends on $C$ and can be estimated from above (for sufficiently large $C \geq C_{0}$ ) in the following way:

$$
\begin{aligned}
1 \leq \ell \leq\left|\beta^{\ell}\right| & =\sum_{i=0}^{\infty}\left\lfloor\frac{\ell}{C^{i}(i+1)}\right\rfloor=\ell+\sum_{i=1}^{\infty}\left\lfloor\frac{\ell}{C^{i}(i+1)}\right\rfloor \leq \ell+\int_{0}^{\infty} \frac{\ell}{C^{x}(x+1)} \mathrm{d} x \\
& =\ell+[\ell C \operatorname{Ei}(-(x+1) \log (C))]_{x=0}^{\infty} \\
& =\ell-\ell C \operatorname{Ei}(-\log (C)),
\end{aligned}
$$

where Ei denotes the exponential integral function, see e.g. 13 for the definition and properties. Since

$$
x \operatorname{Ei}(-\log (x)) \sim-\frac{1}{\log (x)} \quad \text { as } x \rightarrow \infty,
$$

for any $\varepsilon>0$, we can pick $C_{0}$ so that for $C>C_{0}$, we have $-C \operatorname{Ei}(-\log (C))<\varepsilon$. Putting this together, we get

$$
\left|\beta^{\ell}\right| \leq(1+\varepsilon) \ell
$$

for any $\ell$ and $C>C_{0}$ which is (9).

Let $W$ denote the Lambert $W$-function (again, see [13]). Then, using Stirling's formula, we can estimate

$$
\begin{aligned}
& \log \left(C^{\left\|\beta^{\ell}\right\|} \beta^{\ell !}\right)=\sum_{i=0}^{\infty} \log \left(C^{\left\lfloor\frac{\ell}{C^{i}(i+1)}\right\rfloor(i+1)}\left\lfloor\frac{\ell}{C^{i}(i+1)}\right\rfloor !(i+1)^{\left\lfloor\frac{\ell}{C^{i}(i+1)}\right\rfloor}\right) \\
& \left\lfloor\frac{W(\ell C \log (C))}{\log (C)}-1\right\rfloor \\
& =\sum_{i=0}\left\lfloor\frac{\ell}{C^{i}(i+1)}\right\rfloor(i+1) \log (C)+\log \left(\left\lfloor\frac{\ell}{C^{i}(i+1)}\right\rfloor !\right)+\left\lfloor\frac{\ell}{C^{i}(i+1)}\right\rfloor \log (i+1) \\
& \left\lfloor\frac{W(\ell C \log (C))}{\log (C)}-1\right\rfloor \\
& \geq \sum_{i=0}\left\lfloor\frac{\ell}{C^{i}(i+1)}\right\rfloor\left((i+1) \log (C)+\log \left(\left\lfloor\frac{\ell}{C^{i}(i+1)}\right\rfloor\right)-1+\log (i+1)\right) \\
& \left\lfloor\frac{W(\ell C \log (C))}{\log (C)}-1\right\rfloor \\
& \geq \sum_{i=0}^{\log (C)}\left\lfloor\frac{\ell}{C^{i}(i+1)}\right\rfloor\left(\log \left(C^{i+1}(i+1)\left\lfloor\frac{\ell}{C^{i}(i+1)}\right\rfloor\right)-1\right) .
\end{aligned}
$$

Since $\left\lfloor\frac{\ell}{C^{i}(i+1)}\right\rfloor=k$ for some $k \geq 1$ when $i \in\left\{0, \ldots,\left\lfloor\frac{W(\ell C \log (C))}{\log (C)}-1\right\rfloor\right\}$ and hence $\frac{\ell}{C^{i}(i+1)}<k+1$, we see that $\frac{C \ell}{k+1}<C^{i+1}(i+1)$ and hence, using (9), we can estimate $C^{i+1}(i+1)\left\lfloor\frac{\ell}{C^{i}(i+1)}\right\rfloor>\frac{C \ell}{k+1} k \geq \frac{C \ell}{2}=C^{\prime} 2(1+\varepsilon) \ell \geq C^{\prime} 2\left|\beta^{\ell}\right|$ where $C^{\prime}=C /(4(1+\varepsilon))$. Then (11) can be estimated from below by

$$
\begin{aligned}
& \left\lfloor\frac{W(\ell C \log (C))}{\log (C)}-1\right\rfloor \\
& \text { (11) } \geq \sum_{i=0}\left\lfloor\frac{\ell}{C^{i}(i+1)}\right\rfloor\left(\log \left(2\left|\beta^{\ell}\right|\right)+\log \left(C^{\prime}\right)-1\right)=\left|\beta^{\ell}\right|\left(\log \left(2\left|\beta^{\ell}\right|\right)+\log \left(C^{\prime}\right)-1\right) \text {. }
\end{aligned}
$$


This means that, again appealing to Stirling's formula,

$$
C^{2\left\|\beta^{\ell}\right\|}\left(\beta^{\ell !}\right)^{2} \geq\left(\frac{2\left|\beta^{\ell}\right| C^{\prime}}{e}\right)^{2\left|\beta^{\ell}\right|} \geq C^{\prime \prime 2\left|\beta^{\ell}\right|}\left(2\left|\beta^{\ell}\right|\right) !,
$$

where $C^{\prime \prime}=C^{\prime} / e=C /(4 e(1+\varepsilon))$, which is (10).

\section{Estimates on the reduced order factorial of general polyindices}

In this section, we prove a result, Corollary 6.3, which we need to control the order factorials in the commutator formula (5) from Theorem 4.1. In Section [5] we took care of a special case in Lemma 5.1. Here, we show that we can split the general case into two factors, one which is handled by Lemma 5.1, and another, which can be handled by a simple estimate.

Lemma 6.1. There exists a constant $C_{0}>0$ such that for all $C>C_{0}$ and all polyindices $\beta, b$,

$$
c^{\left|\gamma_{\beta+b}\right|} \gamma_{\beta+b} ! \leq C^{\|\beta\|+\|b\|} \beta ! b !,
$$

where $c$ can be chosen as $C /(8 e)$.

Remark 6.2. Note that the constant $c$ can be chosen arbitrarily large as long as $C$ is adjusted accordingly. Note also that Lemma 6.1 is "sharp" in the sense that for all $k$ and all multiindices $\gamma$ with $|\gamma|=k$, and $\beta$ given by $\beta(0)=\gamma$ and $\beta_{\sigma}(i)=0$ for $i \geq 1$ and all $\sigma$, one has that $\|\beta\|=k$ and $\gamma !=\gamma_{\beta} !=\beta$ !.

Proof. We begin by observing that since we can factorize according to dimension $c^{\left|\gamma_{\beta+b}\right|} \gamma_{\beta+b} !=\prod_{\sigma=1}^{d} c^{\left|\beta_{\sigma}\right|+\left|b_{\sigma}\right|}\left(\left|\beta_{\sigma}\right|+\left|b_{\sigma}\right|\right) !$ and $C^{\|\beta\|+\|b\|} \beta ! b !=\prod_{\sigma=1}^{d} C^{\left\|\beta_{\sigma}\right\|+\left\|b_{\sigma}\right\|} \beta_{\sigma} ! b_{\sigma} !$, it is enough to prove that for any pair $\beta, b$ and any $\sigma, 1 \leq \sigma \leq d$, we have

$$
c^{\left|\beta_{\sigma}\right|+\left|b_{\sigma}\right|}\left(\left|\beta_{\sigma}\right|+\left|b_{\sigma}\right|\right) ! \leq C^{\left\|\beta_{\sigma}\right\|+\left\|b_{\sigma}\right\|} \beta_{\sigma} ! b_{\sigma} !,
$$

for some constants $c$ and $C$. Let $\beta, b$ and $\sigma$ be given, let $0<\varepsilon<1$ and $C>C_{0}$ with $C_{0}$ as in Lemma [5.1] and let $\ell \in \mathbb{N}$ be the largest number such that $2\left|\beta^{\ell}\right| \leq\left|\beta_{\sigma}\right|+\left|b_{\sigma}\right|$. Rewrite

$$
\begin{aligned}
C^{\left\|\beta_{\sigma}\right\|+\left\|b_{\sigma}\right\|} \beta_{\sigma} ! b_{\sigma} ! & =\prod_{i=0}^{\infty} C^{\left(\beta_{\sigma}(i)+b_{\sigma}(i)\right)(i+1)} \beta_{\sigma}(i) !(i+1)^{\beta_{\sigma}(i)} b_{\sigma}(i) !(i+1)^{\beta_{\sigma}(i)} \\
& =\prod_{i=0}^{\infty}\left(\prod_{y=1}^{\beta_{\sigma}(i)} C^{(i+1)} y(i+1)\right)\left(\prod_{y=1}^{b_{\sigma}(i)} C^{(i+1)} y(i+1)\right)=\prod_{j=1}^{\left|\beta_{\sigma}\right|+\left|b_{\sigma}\right|} p_{j},
\end{aligned}
$$

for some $p_{j} \leq p_{j+1}$, i.e. a product of $\left|\beta_{\sigma}\right|+\left|b_{\sigma}\right|$ factors of the form $p_{j}=C^{i+1} y(i+1)$ with either $y \leq \beta_{\sigma}(i)$ or $y \leq b_{\sigma}(i)$. Replacing $\beta_{\sigma}$ and $b_{\sigma}$ by $\beta^{\ell}$ in the above identity 
yields

$$
C^{2\left\|\beta^{\ell}\right\|}\left(\beta^{\ell !}\right)^{2}=\prod_{i=0}^{\infty}\left(\prod_{y=1}^{\left\lfloor\frac{\ell}{C^{i}(i+1)}\right\rfloor} C^{i+1} y(i+1)\right)^{2},
$$

where, for each $i, y$ runs through exactly those integers for which $C^{i+1} y(i+1) \leq C \ell$. Note that (14) contains exactly $2\left|\beta^{\ell}\right|$ (non-trivial) factors. We will now compare the first $2\left|\beta^{\ell}\right|$ factors in (13) with the factors appearing in (14). More precisely, we split the ordered product in (13) into those which also appear in (14) and a remainder. Note that by the above discussion this splitting corresponds to sorting the relevant $p_{j}$ into those less than or equal to $C \ell$ and those strictly larger than $C \ell$. The first group can be written as

$$
\prod_{p_{j} \leq C \ell} p_{j}=\prod_{i=0}^{\infty}\left(\prod_{y=1}^{\beta_{\sigma}(i) \wedge \beta^{\ell}(i)} C^{(i+1)} y(i+1)\right)\left(\prod_{y=1}^{b_{\sigma}(i) \wedge \beta^{\ell}(i)} C^{(i+1)} y(i+1)\right),
$$

where $f \wedge g$ denotes the minimum of $f$ and $g$ and the whole product can then be rewritten as in (15).

$$
\begin{aligned}
\prod_{j=1}^{2\left|\beta^{\ell}\right|} p_{j} & =\left(\prod_{i=0}^{\infty}\left(\prod_{y=1}^{\beta_{\sigma}(i) \wedge \beta^{\ell}(i)} C^{(i+1)} y(i+1)\right)\left(\prod_{y=1}^{b_{\sigma}(i) \wedge \beta^{\ell}(i)} C^{(i+1)} y(i+1)\right)\right) \prod_{\substack{j=1 \\
p_{j}>C \ell}}^{2\left|\beta^{\ell}\right|} p_{j} \\
& \geq C^{\prime \prime 2\left|\beta^{\ell}\right|}\left(2\left|\beta^{\ell}\right|\right) ! .
\end{aligned}
$$

To obtain the estimate (16) simply note that for each term missing to obtain $C^{2\left\|\beta^{\ell}\right\|} \beta^{\ell}$ ! in the first product we find one term in the remainder for which $p_{j}>C \ell$. Since the missing term's value must be less than or equal to $C \ell$, we may estimate this $p_{j}$ from below by this missing value. The claimed inequality then follows from (10) in Lemma 5.1 .

Since $\ell$ was chosen as the largest integer such that $2\left|\beta^{\ell}\right| \leq\left|\beta_{\sigma}\right|+\left|b_{\sigma}\right|$, we have that $\left|\beta_{\sigma}\right|+\left|b_{\sigma}\right|<2\left|\beta^{\ell+1}\right| \leq 2(1+\varepsilon)(\ell+1)$, where we for the last inequality used (9) from Lemma 5.1. This implies that $C^{\prime \prime}\left(\left|\beta_{\sigma}\right|+\left|b_{\sigma}\right|\right)<C \ell$. For the remaining $p_{j}$, we then note that

$$
\prod_{j=2\left|\beta^{\ell}\right|+1}^{\left|\beta_{\sigma}\right|+\left|b_{\sigma}\right|} p_{j} \geq(C \ell)^{\left|\beta_{\sigma}\right|+\left|b_{\sigma}\right|-2\left|\beta^{\ell}\right|}>C^{\prime \prime\left|\beta_{\sigma}\right|+\left|b_{\sigma}\right|-2\left|\beta^{\ell}\right|}\left(\left|\beta_{\sigma}\right|+\left|b_{\sigma}\right|\right)_{\left|\beta_{\sigma}\right|+\left|b_{\sigma}\right|-2\left|\beta^{\ell}\right|},
$$

where $(x)_{n}=x(x-1) \cdots(x-(n-1))$ denotes the falling factorial. Putting (16) and (17) together yields (12) with $c=C^{\prime \prime}$.

Corollary 6.3. With $c$ and $C$ as in Lemma 6.1, we have the following estimate:

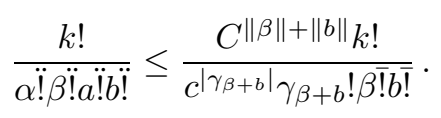

Proof. This follows easily from Lemma 6.1. 


\section{The size of the summation index set}

In this section, we show that the number of terms in the commutator formula (5) from Theorem 4.1 grows in a controllable way.

Proposition 7.1. The number of terms in the iterated commutator formula for the interaction term (5) from Theorem 4.1 is bounded by $c_{d}^{k}$, where $c_{d}$ is some constant which only depends on the dimension $d$.

Proof. For any fixed $k$, the set

$$
\left\{(\alpha, \beta, a, b): \alpha, a \in C_{0}\left(\mathbb{N}_{0}, \mathbb{N}_{0}\right), \beta, b \in C_{0}\left(\mathbb{N}_{0}, \mathbb{N}_{0}^{d}\right),\|\alpha\|+\|\beta\|+\|a\|+\|b\|=k\right\},
$$

where $C_{0}\left(\mathbb{N}_{0}, \mathbb{N}_{0}\right)$ and $C_{0}\left(\mathbb{N}_{0}, \mathbb{N}_{0}^{d}\right)$ denote the sets of 1 - and $d$-dimensional polyindices, respectively, is the index set for the summation formula (5) from Theorem 4.1 for the $k$ 'th iterated commutator of the interaction term $T_{0}$ with the conjugate operator $A$. It can also be written as

$$
\begin{gathered}
\left\{(\alpha, \beta, a, b): \alpha, a, \beta_{\sigma}, b_{\sigma} \in C_{0}\left(\mathbb{N}_{0}, \mathbb{N}_{0}\right), \beta=\left(\beta_{\sigma}\right)_{\sigma=1}^{d}, b=\left(b_{\sigma}\right)_{\sigma=1}^{d},\right. \\
\left.\|\alpha\|+\|a\|+\sum_{\sigma=1}^{d}\left\|\beta_{\sigma}\right\|+\left\|b_{\sigma}\right\|=k\right\} .
\end{gathered}
$$

For any weak composition (see e.g. [7]) $\sum_{j=1}^{2 d+2} k_{j}^{\prime}=k$ of $k$ with exactly $2 d+2$ terms, there are

$$
\prod_{j=1}^{2 d+2} \#\left\{\alpha \in C_{0}\left(\mathbb{N}_{0}, \mathbb{N}_{0}\right):\|\alpha\|=k_{j}^{\prime}\right\}
$$

different ways of satisfying the condition:

$$
\|\alpha\|=k_{1}^{\prime}, \quad\|a\|=k_{2}^{\prime}, \quad\left\|\beta_{\sigma}\right\|=k_{\sigma+2}^{\prime}, \quad\left\|b_{\sigma}\right\|=k_{\sigma+d+2}^{\prime}, \quad \text { for } \sigma=1, \ldots, d .
$$

By Remark 3.1 the set $\left\{\alpha \in C_{0}\left(\mathbb{N}_{0}, \mathbb{N}_{0}\right):\|\alpha\|=k\right\}$ has exactly $p(k)$ elements.

We now want to rewrite the weak composition $\sum_{j=1}^{2 d+2} k_{j}^{\prime}=k$ of $k$ with exactly $2 d+2$ terms in the following way. Let $k_{j}=\sum_{n=1}^{j} k_{n}^{\prime}$ for $j=1, \ldots, 2 d+1$. Then $k_{1}^{\prime}=k_{1}$,

$$
k_{j}^{\prime}=k_{j}-k_{j-1} \quad \text { for } \quad 2 \leq j \leq 2 d+1,
$$

$k_{2 d+2}^{\prime}=k-k_{2 d+1}$, and $0 \leq k_{j} \leq k_{j+1} \leq k$ for any $j=1, \ldots, 2 d$. This means that any weak $2 d+2$-term composition of $k$ is given by a finite, increasing sequence $\left\{k_{j}\right\}_{j=1}^{2 d+1}$, i.e. satisfying $0 \leq k_{1} \leq k_{2} \leq \cdots \leq k_{2 d} \leq k_{2 d+1} \leq k$. We can now count - and estimate - the number of weak $2 d+2$-term compositions of $k$ by counting the number of ways we can choose the sequence $\left\{k_{j}\right\}_{j=1}^{2 d+1}$ :

$$
\begin{gathered}
\sum_{\substack{k_{1}, k_{2}, \ldots, k_{2 d}, k_{2 d+1} \\
0 \leq k_{1} \leq k_{2} \leq \cdots \leq k_{2 d} \leq k_{2 d+1} \leq k}}=\left(\begin{array}{c}
k+2 d+1 \\
2 d+1
\end{array}\right) \\
=\frac{(k+1)(k+2) \cdots(k+2 d)(k+2 d+1)}{(2 d+1) !}<C_{d}^{k},
\end{gathered}
$$


for some sufficiently large constant $C_{d}>0$. Note that in the first step of (18) we have used that the sequence of positive integers $k_{1} \leq k_{2} \leq \cdots \leq k_{2 d+1}$ with $0 \leq k_{i} \leq k$ can be identified with a strictly increasing sequence $h_{1}<h_{2}<\cdots<h_{2 d+1}$ in a 1 to 1 fashion by putting $h_{i}=k_{i}+i$. However, the number of possible choices for such $h_{i}$ obeying $1 \leq h_{i} \leq k+2 d+1$ is exactly $k+2 d+1$ choose $2 d+1$ as claimed in the first step of (18).

We can now estimate the number of elements in the summation index set from above in the following way:

$$
\begin{aligned}
\#\left\{(\alpha, \beta, a, b): \alpha, a \in C_{0}\left(\mathbb{N}_{0}, \mathbb{N}_{0}\right), \beta, b \in C_{0}\left(\mathbb{N}_{0}, \mathbb{N}_{0}^{d}\right),\|\alpha\|+\|\beta\|+\|a\|+\|b\|=k\right\} \\
\quad=\sum_{1} p\left(k_{1}\right) p\left(k_{2}-k_{1}\right) \cdots p\left(k_{2 d+1}-p_{2 d}\right) p\left(k-k_{2 d+1}\right) \\
0 \leq k_{1} \leq k_{2} \leq \cdots \leq k_{2 d} \leq k_{2 d+1} \leq k \\
\quad<\sum e^{c\left(\sqrt{k_{1}}+\sum_{j=2}^{2 d+1} \sqrt{k_{j}-k_{j-1}}+\sqrt{k-k_{2 d+1}}\right)} \\
0 \leq k_{1} \leq k_{2} \leq \cdots \leq k_{2 d} \leq k_{2 d+1} \leq k \\
\leq \sum^{c k} e^{c k} \\
0 \leq k_{1} \leq k_{2} \leq \cdots \leq k_{2 d} \leq k_{2 d+1} \leq k \\
<\left(C_{d} e^{c}\right)^{k}
\end{aligned}
$$

where we used (18) for the last inequality.

\section{Commutators with the free Hamiltonian}

In this section we assume that $V=0$ so that $H(\xi)=H_{0}(\xi)$ is simply given by multiplication with the function $\omega_{\xi}$. Since $A=\frac{i}{2} \operatorname{div}\left(v_{\xi}\right)+\mathfrak{i} v_{\xi} \cdot \nabla_{k}$, it is easy to see that the commutator form $\left[A, M_{f}\right]$, where $f$ is some function, is given by the operator

$$
\operatorname{ad}_{A}\left(M_{f}\right)=M_{\mathfrak{i} v_{\xi} \cdot \nabla_{k} f}
$$

If the gradient is finite almost everywhere, this operator is again just a multiplication operator by a bounded function and is thus bounded as well. In particular, this is true for the choice $f=\omega_{\xi}$, see Section 2 . Furthermore, we may iterate the preceeding calculation and obtain that the $n$ 'th commutator form is given by the bounded multiplication operator

$$
\operatorname{ad}_{A}^{n}\left(M_{f}\right)=M_{\left(\mathfrak{i} v_{\xi} \cdot \nabla_{k}\right)^{n} f}, \quad n \geq 1
$$

provided the $n$-th derivatives of $\mathrm{f}$ remain finite. As noted above the choice $f=\omega_{\xi}$ thus yields a bounded operator.

Proposition 8.1 (Commutator Bounds in the Free Case). For all $n \in \mathbb{N}$ the iterated commutator $\operatorname{ad}_{A}^{n}\left(H_{0}(\xi)\right)$ is given by a bounded multiplication operator as follows

$$
\operatorname{ad}_{A}^{n}\left(H_{0}(\xi)\right)=M_{\left(\mathfrak{i} v_{\xi} \cdot \nabla_{k}\right)^{n} \omega_{\xi}}
$$


where $M_{f}$ is the operator given by multiplication with $f$, and there exists a constant $C_{\xi}>0$ independent of $n$ and $h$, which depends continuously on $\xi \in \mathbb{R}^{d}$ such that we have the pointwise estimate

$$
\left|\left(\mathfrak{i} v_{\xi} \cdot \nabla_{k}\right)^{n} \omega_{\xi}(h)\right| \leq C_{\xi}^{n} n !\langle h\rangle^{2 s_{2}} e^{-h^{2}}
$$

for all $h \in \mathbb{R}^{d}$. In particular, there exists a constant $c_{\xi}$ which depends continuously on $\xi$ such that for all $k \in \mathbb{N}$,

$$
\left\|\operatorname{ad}_{A}^{k}\left(H_{0}(\xi)\right)\right\| \leq c_{\xi}^{k} k !
$$

ProOF. (20) follows directly from (19) with the choice $f=\omega_{\xi}$ and (22) is implied by (21). It thus suffices to prove (21). Note that

$$
\left(v_{\xi} \cdot \mathfrak{i} \nabla_{k}\right)^{n} \omega_{\xi}(k)=\left.\frac{\mathrm{d}^{n-1}}{\mathrm{~d}^{n-1} s} u_{\xi}^{s}(k)\right|_{s=0}, \quad u_{\xi}^{s}(k):=\left(v_{\xi} \cdot \mathfrak{i} \nabla_{k} \omega_{\xi}\right)\left(\gamma_{s}(k)\right)
$$

where $\gamma_{s}$ solves the ODE

$$
\frac{\mathrm{d}}{\mathrm{d} s} \gamma_{s}^{\xi}(k)=v_{\xi}\left(\gamma_{s}^{\xi}(k)\right), \quad \gamma_{0}(k)=k
$$

By Lemma 3.5 of [3], for all $k, \xi \in \mathbb{R}^{d}$ the map $s \mapsto \gamma_{s}^{\xi}(k)$ extends analytically to a strip of some width $r>0$ independent of $k$ and $\xi$, such that $S_{r} \ni z \mapsto \gamma_{z}^{\xi}(k) \in S_{R}^{d}$. Moreover, there exists a constant $C_{\omega}>0$, which is also independent of $k, \xi \in \mathbb{R}^{d}$ such that $\left|\gamma_{z}^{\xi}(k)-k\right| \leq C_{\omega}|z|$, see the Remark 3.8 of [3]. Thus, we may use Cauchy's integral formula to calculate

$$
\begin{aligned}
\left|\left(v_{\xi} \cdot \mathfrak{i} \nabla_{k}\right)^{n} \omega_{\xi}(h)\right| & =\left|\frac{\mathrm{d}^{n-1}}{\mathrm{~d}^{n-1} s} u_{\xi}^{s}(h)\right|_{s=0}|=| \frac{(n-1) !}{2 \pi \mathfrak{i}} \int_{\Gamma_{r}} \frac{u_{\xi}\left(\gamma_{z}^{\xi}(h)\right)}{z^{n}} \mathrm{~d} z \mid \\
& \leq(n-1) ! \frac{\sup _{z \in \Gamma_{r}}\left|u_{\xi}\left(\gamma_{z}^{\xi}(h)\right)\right|}{r^{n-1}}
\end{aligned}
$$

where $\Gamma_{r}$ denotes the set $\{|z|=r\}$, and, by abuse of notation, a path parametrizing this set in the counter-clockwise direction. By applying Peetre's inequality,

$$
\forall q \in \mathbb{R}, k, h \in \mathbb{C}^{d}: \quad\langle k+h\rangle^{q} \leq 2^{|q|}\langle k\rangle^{|q|}\langle h\rangle^{q},
$$

see e.g. [15, Lemma 1.18], the assumptions, and the estimate $\left|\gamma_{z}^{\xi}(k)-k\right| \leq C_{\omega}|z|$, we may estimate

$$
\sup _{z \in \Gamma_{r}}\left|u_{\xi}\left(\gamma_{z}^{\xi}(h)\right)\right| \leq \tilde{C}_{\xi}\langle h\rangle^{2 s_{2}} e^{-h^{2}}
$$

for some constant $\tilde{C}_{\xi} \geq 1$, which can be chosen such that it depends continuously on $\xi \in \mathbb{R}^{d}$. Since $\tilde{C}_{\xi} \geq 1$, we may define $C_{\xi}=\frac{\tilde{C}_{\xi}}{\max \{1, r\}}$ and conclude the statement. 


\section{Estimates on the interaction commutator}

In this section, we make estimates on the commutator from Theorem 4.1, using the estimates established in Sections 5 and 6 on the order factorials, and in Section 7 on the number of terms. We also use Proposition 8.1 from Section 8 to control the multiplication operators $M_{\alpha, \beta}$.

Lemma 9.1. There exists a constant $C_{\xi}^{\prime}$ which depends continuously on $\xi \in \mathbb{R}^{d}$ such that for all $h \in \mathbb{R}^{d}$, all $n \in \mathbb{N}$, and all $\sigma \in\{1,2, \ldots, d\}$, we have the pointwise estimate

$$
\left|\left(\mathfrak{i} v_{\xi} \cdot \nabla\right)^{n} v_{\xi, \sigma}(h)\right| \leq C_{\xi}^{\prime n} n !\langle h\rangle^{2 s_{2}} e^{-h^{2}} .
$$

Proof. Mimic the proof of Proposition 8.1 with $\omega_{\xi}$ replaced by $v_{\xi, \sigma}$.

Proposition 9.2 (Estimates on $M_{\alpha, \beta}$ ). Let $\alpha$ and $\beta$ be 1 - and d-dimensional polyindices, respectively, and write $f_{\alpha, \beta}=\mathbb{D}_{v} w^{\alpha} \mathbb{D}_{v} v_{\xi}^{\beta}$, such that $M_{\alpha, \beta}=M_{f_{\alpha, \beta}}$. Then we have the following pointwise estimate

$$
\left|f_{\alpha, \beta}(h)\right| \leq C_{\xi}^{\prime \prime|| \alpha\|+\| \beta \|}\left(\langle h\rangle^{2 s_{2}} e^{-h^{2}}\right)^{|\alpha|+|\beta|} \alpha \overline{!} \beta \overline{!},
$$

where $C_{\xi}^{\prime \prime}=\max \left\{C_{\xi}, C_{\xi}^{\prime}\right\}$ and $C_{\xi}$ and $C_{\xi}^{\prime}$ are the constants from Proposition 8.1] and Lemma 9.1, respectively.

ProOF. Note that $M_{\alpha, \beta}=M_{\mathbb{D}_{v} w^{\alpha}} M_{\mathbb{D}_{v} v_{\xi}^{\beta}}$, and that we may write

$$
M_{\mathbb{D}_{v} w^{\alpha}}=\prod_{i=0}^{\infty} M_{\left(D_{v}^{i} w\right)^{\alpha(i)}}
$$

Put $w_{\xi}^{0}(k):=w(k)$ and note that $\left(\left(\mathfrak{i} v_{\xi} \cdot \nabla_{k}\right)^{n} \omega_{\xi}\right)(k)=\left(\left(\mathfrak{i} v_{\xi} \cdot \nabla_{k}\right)^{n-1} w_{\xi}\right)(k)$. We now use Proposition 8.1 to get the pointwise estimate

$$
\begin{aligned}
\left|\mathbb{D}_{v} w^{\alpha}(h)\right| & =\prod_{i=0}^{\infty}\left|\left(\left(D_{v}^{i} w\right)(h)\right)^{\alpha(i)}\right| \leq \prod_{i=0}^{\infty}\left(C_{\xi}^{i} i !\langle h\rangle^{2 s_{2}} e^{-h^{2}}\right)^{\alpha(i)} \\
& \leq C_{\xi}^{\|\alpha\|}\left(\langle h\rangle^{2 s_{2}} e^{-h^{2}}\right)^{|\alpha|} \prod_{i=0}^{\infty} i !^{\alpha(i)} .
\end{aligned}
$$

Likewise, we note that

$$
M_{\mathbb{D}_{v} v_{\xi}^{\beta}}=\prod_{\sigma=1}^{d} \prod_{i=0}^{\infty} M_{\left(D_{v}^{i} v_{\xi, \sigma}\right)^{\beta \sigma(i)}}
$$

which we use to compute the pointwise estimate as before

$$
\left|M_{\mathbb{D}_{v} v_{\xi}^{\beta}}(h)\right| \leq C_{\xi}^{\prime\|\beta\|}\left(\langle h\rangle^{2 s_{2}} e^{-h^{2}}\right)^{|\beta|} \prod_{\sigma=1}^{d} \prod_{i=1}^{\infty} i !^{\beta_{\sigma}(i)} .
$$

Combining these two estimates now gives the proposition. 
Proposition 9.3. If for all d-dimensional polyindices $\beta, b \in C_{0}\left(\mathbb{N}_{0}, \mathbb{N}_{0}^{d}\right)$ with total order less than $k,\|\beta\|+\|b\| \leq k$, the forms

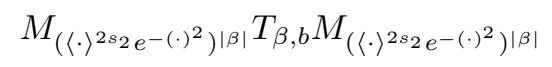

on $C_{0}^{\infty}\left(\mathbb{R}^{d}\right)$ extend to bounded operators on $\mathrm{L}^{2}\left(\mathbb{R}^{d}\right)$, then so do the forms in (5) from Theorem 4.1 (with $M_{\alpha, \beta} T_{\beta, b} M_{a, b}$ interpreted as the bounded operator given by the form). Furthermore, we have the following estimate on $\operatorname{ad}_{A}^{k}\left(T_{0}\right)\left(H_{0}(\xi)+\mathfrak{i}\right)^{-1}$ :

$$
\begin{aligned}
& \left\|\operatorname{ad}_{A}^{k}\left(T_{0}\right)\left(H_{0}(\xi)+\mathfrak{i}\right)^{-1}\right\|
\end{aligned}
$$

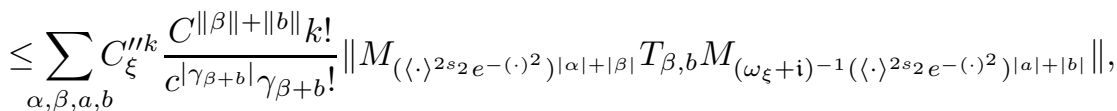

where $C_{\xi}^{\prime \prime}$ is the constant from Proposition 9.2, which depends continuously on $\xi$, and $c$ and $C$ are the constants from Lemma 6.1.

Proof. This follows easily from Theorem 4.1, Theorem 6.3, and Proposition 9.2 ,

\section{Theorem 9.4.}

1. Assume that for some $c>0$ and all pairs of polyindices $\beta, b$,

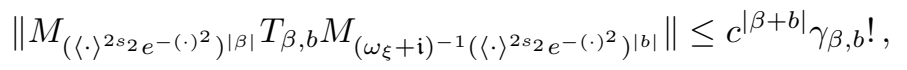

where $\gamma_{\beta, b}$ is given as in (44), and $M_{\left(\langle\cdot\rangle^{2} s_{2} e^{\left.-(\cdot)^{2}\right)^{|\beta|}}\right.} T_{\beta, b} M_{\left(\omega_{\xi}+\mathfrak{i}\right)^{-1}\left(\langle\cdot)^{2} s_{2} e^{-(\cdot)^{2}}\right)^{|b|}}$ is interpreted as the bounded operator given by the form on $C_{0}^{\infty}\left(\mathbb{R}^{d}\right)$. Then there exists a constant $C>1$ such that

$$
\left\|\operatorname{ad}_{A}^{k}\left(T_{0}\right)\left(H_{0}(\xi)+\mathfrak{i}\right)^{-1}\right\| \leq\left(C C_{\xi}^{\prime \prime} c_{d}\right)^{k} k !
$$

and the constant $C_{\xi}^{\prime \prime}$, which depends continuously on $\xi$, comes from Proposition 9.2 and $c_{d}$ comes from Proposition 7.1 .

2. In particular, (24) holds, if there exists a constant $c>0$ such that for all $\beta, b$,

$$
\left\|T_{\beta, b}\left(H_{0}(\xi)+\mathfrak{i}\right)^{-1}\right\| \leq c^{|\beta+b|} \gamma_{\beta, b} !
$$

3. Suppose that $V$ is as in either Condition 2.2 or Condition 2.4. Then (25) is satisfied.

Proof. The first part of the statement follows immediately from Remark 6.2. Proposition 7.1 and Proposition 9.3 . The second statement follows from observing that if $\left\|T_{\beta, b}\left(H_{0}(\xi)+\mathfrak{i}\right)^{-1}\right\|<\infty$, then

$$
\left\|M_{\left(\langle\cdot\rangle^{2} s_{2} e^{-(\cdot)^{2}}\right)^{|\beta|}} T_{\beta, b} M_{\left(\omega_{\xi}+\mathfrak{i}\right)^{-1}\left(\langle\cdot)^{2 s_{2}} e^{-(\cdot)^{2}}\right)^{|b|}}\right\| \leq c_{M}^{|\beta+b|}\left\|T_{\beta, b}\left(H_{0}(\xi)+\mathfrak{i}\right)^{-1}\right\|
$$


for some $C_{M}>0$. The third statement can be seen to be correct by the following argument. Let $\hat{V}$ satisfy Condition 2.2. We introduce the shorthand $j_{p}(k):=(1+$ $\left.\langle k\rangle^{p}\right)^{-1}$, where $p:=s_{2}$, see Condition 2.1. By the weak Young inequality, see [10, p. 107],

$$
\begin{aligned}
\left\|T_{\beta, b}\left(H_{0}(\xi)+\mathfrak{i}\right)^{-1}\right\| & =\sup _{\substack{\phi, \psi \in \mathcal{H} \\
\|\phi\|=\|\psi\|=1}} \mid\left\langle\phi, T_{\beta, b}\left(H_{0}(\xi)+\mathfrak{i}\right)^{-1} \psi\right\rangle . \\
\leq\left\|\hat{V}^{\left(\gamma_{\beta, b}\right)}\right\|_{s, w} \sup _{\substack{\psi \in \mathcal{H} \\
\|\psi\|=1}}\left\|j_{p} \psi\right\|_{t} &
\end{aligned}
$$

where $\frac{1}{s}+\frac{1}{t}=\frac{3}{2}$. Due to $s \in(1,2), t \in(1,2)$. Now by Hölder's inequality

$$
\left\|j_{p} \psi\right\|_{t} \leq\|\psi\|_{2}\left\|j_{p}^{t}\right\|_{\frac{2}{2-t}}^{\frac{1}{t}}
$$

which is finite, if $-p \frac{2 t}{2-t}+d<0$. This however is equivalent to $p>d\left(1-\frac{1}{s}\right)$ which is true by assumption. Thus,

$$
\left\|T_{\beta, b}\left(H_{0}(\xi)+\mathfrak{i}\right)^{-1}\right\| \leq\left\|\hat{V}^{\left(\gamma_{\beta, b}\right)}\right\|_{s, w}\left\|j_{p}^{t}\right\|_{\frac{2}{2-t}}^{\frac{1}{t}}
$$

and we see that the third statement follows if we assume Condition 2.2. If $\mathrm{V}$ satisfies Condition 2.4, the proof is similar and can be carried out directly by applying (the ordinary) Young inequality.

\section{The Yukawa potential}

Before proving Proposition 2.7 we will introduce some notation. Let $d \in \mathbb{N}$ and $D_{1}, \ldots, D_{d}$ be discs in $\mathbb{C}$ of radius $r$. We then define $D:=D_{1} \times \cdots \times D_{d}$. We denote by $\Gamma$ the distinguished boundary of $D$, that is $\Gamma=\partial D_{1} \times \cdots \times \partial D_{d}$. Moreover, for any $\alpha \in \mathbb{N}^{d}$ and $z \in \mathbb{C}^{d}$ we define $z^{\alpha}:=\prod_{j=1}^{d} z_{j}^{\alpha_{j}}$. For an analytic function $f: U \subset \mathbb{C}^{d} \rightarrow \mathbb{C}^{d}$ we denote by $f^{(\alpha)}$ the iterated partial derivatives of $f$ corresponding to the multi-index $\alpha$, that is $\alpha_{j}$ derivatives w.r.t. the $j$-th variable. If we denote by $\alpha+\mathbf{1} \in \mathbb{N}^{d}$ the multi-index with whose $j$-th coordinate is $\alpha_{j}+1$, the $d$-dimensional generalization of Cauchy's formula is then

$$
f^{(\alpha)}(z)=\int_{\Gamma} \frac{f(w)}{(z-w)^{\alpha+1}} \mathrm{~d}^{d} w
$$

Having taken care of these notational issues we can provide a proof of Proposition 2.7

Proof of Proposition 2.7. Clearly $\hat{V}$ has an extension to an analytic function into the 3-dimensional strip $S_{1}^{3}:=\left\{z \in \mathbb{C}^{3}|| \operatorname{Im}\left(z_{j}\right) \mid<\tilde{r}\right\}$, where $\tilde{r}<1$. Hence, for $r \in(0, \tilde{r})$ 
and $k \in \mathbb{R}^{3}$ the 3-dimensional Cauchy formula allows us to estimate

$$
\begin{aligned}
\left|\hat{V}^{(\alpha)}(k)\right| & \leq \frac{\alpha !}{(2 \pi)^{3}}\left|\int_{\Gamma} \frac{\hat{V}(z)}{(k-z)^{\alpha+1}} \mathrm{~d} z\right| \leq \frac{\alpha !}{(2 \pi)^{3}} \frac{1}{r^{|\alpha|}} \int_{0}^{2 \pi} \int_{0}^{2 \pi} \int_{0}^{2 \pi} \frac{4 \pi}{1+\left.|r-| k\right|^{2}} \mathrm{~d} t_{1} \mathrm{~d} t_{2} \mathrm{~d} t_{3} \\
& =\frac{\alpha !}{r^{|\alpha|}} \frac{4 \pi}{1+|r-| k||^{2}},
\end{aligned}
$$

where $\Gamma$ is the distinguished boundary of the 3 dimensional polydisc of radius $r$. Let $\beta>0$. By the above computations we thus have

$$
\begin{aligned}
\left\{k \in \mathbb{R}^{3}|| \hat{V}^{(\alpha)}(k) \mid>\beta\right\} & \subset\left\{k \in \mathbb{R}^{3} \mid \frac{\alpha !}{r^{|\alpha|}} \frac{4 \pi}{1+\left.|r-| k\right|^{2}}>\beta\right\} \\
& =\left\{k \in \mathbb{R}^{3}\left|r-\left(\frac{1}{\beta_{0}}-1\right)^{\frac{1}{2}}<\right| k \mid<\left(\frac{1}{\beta_{0}}-1\right)^{\frac{1}{2}}+r\right\} \\
& \subset\left\{k \in \mathbb{R}^{3}|| k \mid<\left(\frac{1}{\beta_{0}}-1\right)^{\frac{1}{2}}+r\right\},
\end{aligned}
$$

where $\beta_{0}=\frac{r^{|\alpha|}}{\alpha ! 4 \pi} \beta$. Due to $\left\{k \in \mathbb{R}^{3} \mid\left(1+|r-| k||^{2}\right)^{-1}>\beta\right\}=\emptyset$ for $\beta \geq 1$, we can use the above inclusions to compute

$$
\begin{aligned}
\left\|\hat{V}^{(\alpha)}\right\|_{s, w} & =\sup _{\beta>0} \beta\left|\left\{k \in \mathbb{R}^{3}|| \hat{V}^{(\alpha)}(k) \mid>\beta\right\}\right|^{\frac{1}{s}} \\
& \leq 4 \pi \frac{\alpha !}{r^{|\alpha|}} \sup _{\beta \in(0,1)} \beta\left|\left\{k \in \mathbb{R}^{3} \mid \frac{1}{1+\left.|r-| z\right|^{2}}>\beta\right\}\right|^{\frac{1}{s}} \\
& \leq 4 \pi \frac{\alpha !}{r^{|\alpha|}} \sup _{\beta \in(0,1)} \beta\left|\left\{k \in \mathbb{R}^{3}|| k \mid<\left(\frac{1}{\beta}-1\right)^{\frac{1}{2}}+r\right\}\right|^{\frac{1}{s}} \\
& =16 \pi^{2} \frac{\alpha !}{r^{|\alpha|}} M_{s},
\end{aligned}
$$

where

$$
M_{s}=\sup _{\beta \in(0,1)} \beta^{1-\frac{3}{2 s}}\left((1-\beta)^{\frac{1}{2}}+r \beta^{\frac{1}{2}}\right)^{\frac{3}{s}}<\infty,
$$

due to $s>3 / 2$. Choosing $c=\max \left\{16 \pi^{2} M_{s}, 1\right\} r^{-1}$ completes the proof of the statement.

\section{Acknowledgments}

The authors would like to thank Jacob Schach Møller for useful discussions. M. E. acknowledges the support of the Lundbeck Foundation and the German Research Foundation (DFG) through the Graduiertenkolleg 1838 and M.G.R. acknowledges support from the Danish Council for Independent Research - Natural Sciences, grant 12124675, "Mathematical and Statistical Analysis of Spatial Data". 


\section{References}

[1] W. O. Amrein, A. Boutet de Monvel, and V. Georgescu, $C_{0}$-groups, commutator methods and spectral theory of N-body Hamiltonians, Birkhäuser, 1996.

[2] L. Cattaneo, G.M. Graf, and W. Hunziker, A general resonance theory based on Mourre's inequality, Ann. Henri Poincaré 7 (2006), 583-601.

[3] M. Engelmann, J. S. Møller, and M. G. Rasmussen, Local spectral deformation, arXiv:1508.03474, 2015, Submitted.

[4] J. Faupin, J. S. Møller, and E. Skibsted, Regularity of bound states, Rev. in Math. Phys. (2011), no. 5, 453-530.

[5] - Second order perturbation theory for embedded eigenvalues, Comm. Math. Phys. 306 (2011), 193-228.

[6] G. H. Hardy and E. M. Wright, An introduction to the theory of numbers, sixth ed., Oxford University Press, 2008, Revised by D. R. Heath-Brown and J. H. Silverman.

[7] S. Heubach and T. Mansour, Combinatorics of compositions and words, Discrete Mathematics and its Applications, CRC Press, 2009.

[8] W. Hunziker and I. M. Sigal, The quantum N-body problem, J. Math. Phys. 41 (2000), 3448-3510.

[9] A. Jensen, E. Mourre, and P. Perry, Multiple commutator estimates and resolvent smoothness in quantum scattering theory, Ann. Inst. Henri Poincaré 41 (1984), no. $2,270-225$.

[10] E. H. Lieb and M. Loss, Analysis, 2nd ed., Graduate Studies in Mathematics, American Mathematical Society, 2001.

[11] J. S. Møller and M. Westrich, Regularity of eigenstates in regular mourre theory, J. Funct. Anal. 260 (2011), 852-878.

[12] E. Mourre, Absence of singular continuous spectrum for certan self-adjoint operators, Comm. Math. Phys. 78 (1981), 391-408.

[13] F. W. J. Olver, D. W. Lozier, R. F. Boisvert, and C. W. Clark (eds.), NIST handbook of mathematical functions, Cambridge University Press, 2010.

[14] M. G. Rasmussen, A taylor-like expansion of a commutator with a function of self-adjoint, pairwise commuting operators, Math. Scand. 111 (2012), no. 1, 107117 .

[15] X. Saint Raymond, Elementary introduction to the theory of pseudodifferential operators, Studies in Advanced Mathematics, CRC Press, Boca Raton, FL, 1991. 